\title{
von Neumann e a descrição da Mecânica Quântica no espaço de fases
}

von Neumann and the phase space description of Quantum Mechanics

\author{
Diógenes Galetti*1@, Marcelo A. Marchiolli² $^{2}$, Celso L. Lima ${ }^{3}$ \\ ${ }^{1}$ Universidade Estadual Paulista, Instituto de Fisica Teorica, São Paulo, SP, Brasil \\ ${ }^{2}$ Pesquisador Autônomo, Jaboticabal, SP, Brasil \\ ${ }^{3}$ Universidade de São Paulo, Instituto de Física, Departamento de Física Nuclear, São Paulo, SP, Brasil
}

\begin{abstract}
Recebido em 23 de Setembro, 2018. Revisado em 13 de Dezembro, 2018. Aceito em 18 de Dezembro, 2018.
Ao fundamentar as bases da Mecânica Quântica através da solução de um problema matemático associado a possíveis inconsistências na relação de comutação para os operadores posição e momento linear, von Neumann lança as bases teóricas do que posteriormente resultou na descrição da Mecânica Quântica no espaço de fases. No presente trabalho, complementar à tradução do texto original de von Neumann, analisamos um conjunto específico de resultados, que formam um arcabouço matemático sólido para a concepção de um espaço de fases genuinamente quântico, tornando-o assim um legítimo precursor desta descrição.
\end{abstract}

Palavras-chave: mecânica quântica; von Neumann; relação de comutação; espaço de fases.

By grounding the foundations of Quantum Mechanics through the solution of a mathematical problem associated with possible inconsistencies in the position and momentum commutation relation, von Neumann provides the theoretical underpinnings of later resulted in the phase space description of Quantum Mechanics. In this work, complementary to the translation of von Neumann's original text, we analyze a specific set of results that constitutes a solid mathematical background for the design of a genuinely quantum phase space, thus making it a legitimate precursor to this description.

Keywords: quantum mechanics; von Neumann; commutation relation; phase space.

\section{Introdução}

O desenvolvimento da Mecânica Quântica (MQ) foi uma necessidade gerada pelo acúmulo de resultados experimentais ao longo da virada dos séculos XIX e XX, os quais não conseguiam ser entendidos e/ou explicados à luz das teorias físicas existentes naquele período. As tentativas de contornar as dificuldades através da adaptação dos formalismos e ferramentas então disponíveis foram paulatinamente abandonadas, pois logo ficou claro que novas frentes conceituais e técnicas teriam que ser abertas. As propostas de uma equação de onda, que generalizava ideias acerca do caráter ondulatório das partículas, bem como de uma formulação matricial, baseada na utilização de observáveis experimentais na descrição dos sistemas atômicos, logo foram seguidas por trabalhos mais marcadamente matemáticos, que tinham por principal objetivo aparar possíveis arestas formais surgidas ao longo desse avanço conceitual.

Também foi árdua e não trivial a tarefa de elaborar um formalismo matemático para a descrição da Mecânica Quântica no espaço de fases, que compatibilizasse, em pé de igualdade, ambas as variáveis do tipo momento linear e posição clássicas com preceitos quânticos importantes,

*Endereço de correspondência: diogaletti@hotmail.com como aquele regido pelo princípio de incerteza de Heisenberg. Esta tarefa foi abraçada por um número razoável de físicos teóricos na primeira metade do século XX [1], cuja soma de esforços [2] forma um intrincado quebra-cabeças culminando no que atualmente é conhecido por formalismo WWGM, devido às contribuições fundamentais da tétrade Weyl-Wigner-Groenewold-Moyal. Ao interpretarmos o formalismo em questão como uma descrição legítima da MQ no espaço de fases, comentar sobre as contribuições de von Neumann para os fundamentos teóricos da MQ é também resgatar, de um ponto de vista histórico, um conjunto de resultados matemáticos que anteveem certas bases importantes para o formalismo WWGM 1

Em particular, vamos focar seu artigo publicado em Mathematische Annalen [4] no ano de 1931, 2] que é considerado um marco importante para o desenvolvimento

\footnotetext{
${ }^{1}$ Informações adicionais acerca das diferentes contribuições de von Neumann para a ciência são encontradas resumidamente na figura 1 em conjunto com alguns dados biográficos pertinentes.

${ }^{2}$ Este trabalho foi escrito originalmente em alemão e a versão traduzida para o português-BR pode ser encontrada neste volume da Revista Brasileira de Ensino de Física (RBEF) como material suplementar.
} 


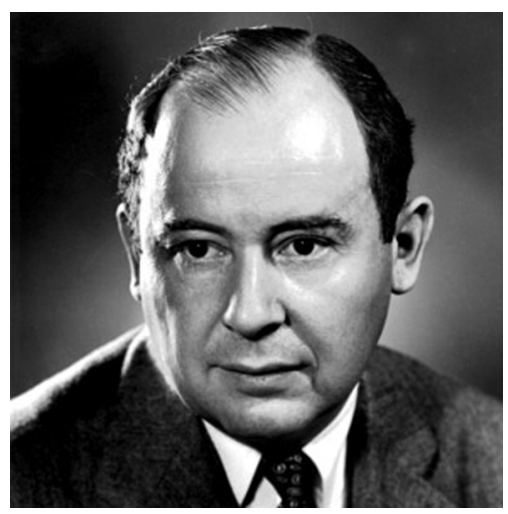

Figura 1: Margittai Neumann János Lajos (nome de origem) e/ou John von Neumann (nome anglicizado): Budapeste, 28 de dezembro de 1903 (nascimento) - Washington, 8 de fevereiro de 1957 (falecimento). Matemático húngaro de origem judaica e naturalizado estadunidense, é considerado por muitos como um dos matemáticos mais importantes do século $\mathrm{XX}$. As suas contribuiç̃̃es são inúmeras, abrangendo as áreas de: teoria dos conjuntos, análise funcional, teoria ergódica, mecânica quântica ${ }^{4}$ ciência da computação, economia, teoria dos jogos, hidrodinâmica de explosões, entre outras. Além disso, foi consultor do Projeto Manhattan, instituido para o desenvolvimento e construção das primeiras bombas atômicas. Esta imagem foi extraída e adaptada do endereço eletrônico: https://www . famousscientists . org/john-von-neumann/.

desse formalismo 3 Antes de darmos início à análise deste trabalho, convém recordarmos alguns fatos importantes pertinentes às descrições mais utilizadas da MQ $\left[\begin{array}{lll}5 & 12\end{array}\right.$ e que paralelizam com a sua descrição no espaço de fases 13 14. Posteriormente descreveremos o conteúdo das demais seções.

\section{A Mecânica Quântica e suas Descrições}

Com a apresentação de uma série de trabalhos sobre os fundamentos matemáticos da MQ 15 21], propostas anteriores, baseadas essencialmente na formulação da mecânica clássica e eletromagnetismo, foram questionadas e seus limites testados $5[$ 8. Neste contexto, não só os conceitos fundamentais da física foram alvo de críticas e reavaliações, como também o caráter matemático desta nova abordagem foi posto em evidência. Com isso, Born e Jordan [16] propuseram a utilização de espaços vetoriais complexos - em particular, espaços de Hilbert ${ }^{5} 22$ 25 -

${ }^{3}$ Por exemplo, ver palestra proferida pelo Prof. Basil Hiley sobre Mecânica Quântica no Espaço de Fases, disponível no endereço eletrônico https://www . youtube. com/watch?v=xT6KVnRhQNc.

${ }^{4} \mathrm{Um}$ resumo das contribuições de von Neumann para a mecânica quântica pode ser encontrado na Ref. 3].

${ }^{5}$ Termo criado por von Neumann em homenagem a David Hilbert. $\mathrm{O}$ conceito de 'espaço de Hilbert' utilizado aqui refere-se àquele conceito matemático que generaliza a noção de espaço euclidiano. Este espaço vetorial abstrato possui a estrutura de um produto interior complexo que permite as noções de comprimento e ângulo serem medidos, daí o seu caráter geométrico. como um substrato matemático para descrever o caráter cinemático da MQ. Dessa forma, este espaço vetorial conteria toda a informação referente aos possíveis estados do sistema físico em discussão. Neste formalismo, o elo com a mecânica das matrizes - inicialmente apresentada por Heisenberg 15 e Born 18,19 - era direto e o problema da descrição de um oscilador harmônico quântico resolvido satisfatoriamente 9 .

Ao mesmo tempo em que eram invocados conceitos referentes a espaços de Hilbert, também era trazido à tona o conceito de operadores atuando sobre os elementos daqueles espaços vetoriais. Por sua vez, estes devem satisfazer condições específicas para representarem observáveis físicos: serem hermitianos. Isto posto, o estudo de suas propriedades matemáticas desempenharia então um papel essencial na sua caracterização completa. De partida, ficou claro o papel de destaque do par de operadores associados à posição e ao momento linear de uma partícula. Nesse novo formalismo da MQ, estes operadores não comutam entre si, fato este transcrito na relação de comutação

$$
[\hat{q}, \hat{p}]=\mathrm{i} \hbar \hat{1} \quad \operatorname{com} \quad \hbar \equiv \frac{h}{2 \pi},
$$

sendo $h$ a constante de Planck e $\hat{1}$ o operador identidade 6

Entrementes, Erwin Schrödinger [27] propõe uma das descrições da MQ mais amplamente aceitas na atualidade. Neste formalismo, todas as partículas são descritas individualmente e/ou coletivamente, no nível microscópico, por uma entidade matemática abstrata definida no corpo dos números complexos, denominada função de onda $\Psi$. Esta função satisfaz, em particular, uma equação diferencial parcial linear de segunda ordem, sendo designada na literatura por equação de Schrödinger [28 30]. Com esta equação, foi possível entender o espectro do átomo de hidrogênio e as transições entre seus níveis de energia. É digno de nota observar que o próprio Schrödinger 31., bem como Eckart [32], mostrou a equivalência entre a sua descrição e aquela de Born-Heisenberg-Jordan [17], tendo ele, para tanto, utilizado a abordagem de Dirac [20].

$\mathrm{O}$ acúmulo de dados experimentais ao longo dos anos mostrou claramente que o comportamento das partículas do mundo microscópico é inerentemente probabilístico. Porém, convém salientar que a equação diferencial na formulação original de Schrödinger não lida diretamente com probabilidades, mas sim com funções de onda. Entretanto, funções de onda e probabilidades foram conectadas por intermédio da interpretação probabilística de Born [18, 19], pela qual a grandeza associada ao quadrado do módulo da função de onda, $|\Psi|^{2}$, é diretamente proporcional à probabilidade de que uma partícula seja encontrada em uma certa região do espaço.

É oportuno mencionar que a interpretação estatística da MQ, gestada em Copenhague, só foi chancelada após

\footnotetext{
${ }^{6}$ Heisenberg menciona a relação de comutação (1) explicitamente em seu trabalho seminal de 1927 [26], cujos resultados culminaram na célebre relação de incerteza para os operadores momento linear e posição, a qual posteriormente recebeu seu nome.
} 
calorosos embates acerca de seus fundamentos teóricos entre os famosos e emblemáticos participantes da quinta Conferência de Solvay, realizada em Bruxelas na Bélgica, nos dias 23-29 de Outubro de 1927.

Numa outra vertente, baseado nas observações de Dirac $33 \quad 35$ sobre uma possível relação da ação clássica 7 com a MQ, Feynman 37] estabelece em 1948 o que seria considerada então uma terceira descrição da MQ, usando agora integrais de trajetória 8 Embora seja uma abordagem conceitual inovadora, já que incorpora os fenômenos de interferência quânticos, sua aplicabilidade imediata possui restrições: não é transparente no que tange à invariância por transformações unitárias, além de apresentar dificuldades técnicas inerentes ao processo de integração funcional ${ }^{9}$ Com o advento da teoria quântica de campos, o formalismo funcional torna-se de imediato uma ferramenta útil e extremamente poderosa [39].

Vamos agora retomar as duas primeiras descrições da MQ e, em particular, discutir a relação de comutação (1), que tem um papel central para elas e serve primordialmente de guia para avalizar a caraterística diferenciada desta nova mecânica com relação à mecânica clássica. Considerações matemáticas associadas a esta relação de comutação motivaram, contudo, a proposição de uma alternativa a ela. Assim, ao preservar a não comutatividade dos operadores momento linear e posição, a nova proposta envolve uma família de operadores unitários que, simultaneamente, servem de fundamento para a elaboração de uma técnica de mapeamento que conecta funções de variáveis canônicas clássicas e operadores da MQ. Este formalismo, desenvolvido por Weyl [40], sugeria um possível processo de quantização, o que posteriormente mostrou-se não ser correto. Apesar de não ser um procedimento de quantização, a estrutura matemática envolvendo a família de operadores unitários foi fundamental para a construção de uma nova descrição da MQ, a assim chamada descrição de espaço de fases. Com isso, operadores estão associados a funções de variáveis canônicas clássicas (e vice-versa), num mapeamento com regras bem estabelecidas e todos os procedimentos matemáticos próprios da MQ têm sua contraparte nesta nova descrição. Consequentemente, ao operador que descreve o estado do sistema físico fica então associada uma função particular (conhecida como função de Wigner), aos demais operadores, suas funções correspondentes e, por último, para a equação de evolução temporal tem-se como contrapartida a chamada equação de Moyal [41].

Embora tenha passado despercebido por muitos autores até então, o trabalho de von Neumann [4] representa um marco crucial no início do desenvolvimento da descrição da MQ no espaço de fases. Nele, o referido autor

\footnotetext{
${ }^{7}$ O termo "ação" corresponde, para uma dada trajetória, ao processo de integração no tempo da lagrangeana associada ao sistema. No caso de uma partícula movendo-se classicamente, a obtenção da trajetória efetivamente seguida, requer o uso do princípio de mínima ação de Hamilton 36.

${ }^{8} \mathrm{Ou}$ integração funcional, como tecnicamente é conhecida.

${ }^{9} \mathrm{O}$ átomo de hidrogênio é o exemplo mais conhecido 38 .
}

abordou o problema matemático concernente à relação de comutação de Weyl para os operadores unitários e, como subproduto, determinou a forma correta de representar um operador como uma função de variáveis canônicas clássicas. Este passo foi fundamental - embora não tenha sido o seu objetivo - para mostrar como estabelecer um mapeamento entre operadores e funções de variáveis canônicas clássicas, bem como o seu reverso, de forma rigorosa, fato este completamente inédito para a época. Além disso, um resultado importante apresentado explicitamente neste trabalho consiste na forma de se determinar a associação entre um produto de dois operadores e suas funções individuais correspondentes: a convolução requerida para tal é o embrião do que mais tarde se chamou produto- $\star$. Por fim, as ideias conjuntas de Weyl e von Neumann abriram um caminho seguro para a descrição da MQ no espaço de fases; os trabalhos de Wigner 42], Groenewold [43] e Moyal 44, 45] pavimentaram as bases dessa descrição.

Procederemos na seção 3 à análise de um conjunto de resultados específicos estabelecidos por von Neumann, que são os precursores do formalismo WWGM. Na seção 4 realizaremos uma discussão sobre suas conexões com a descrição da MQ no espaço de fases, enfatizando detalhes importantes que fundamentam a abordagem adotada nessa descrição. Na seção 5 apresentaremos as nossas considerações finais.

\section{O Trabalho de von Neumann}

Vamos considerar neste momento a abordagem da MQ de Heisenberg-Born-Jordan e, em particular, as críticas expostas por Weyl [40] e von Neumann [4] com relação ao rigor matemático empregado naquela descrição. Ambos apontaram inicialmente para um problema de caráter matemático presente na relação fundamental (1); numa primeira análise, foi observado explicitamente que os operadores coordenada e momento linear não satisfazem as propriedades requeridas para serem operadores bona fide atuando nos elementos de um espaço de Hilbert 10 No caso específico da relação fundamental, observa-se que o lado esquerdo desta equação envolve dois operadores não limitados ( $\hat{q}$ e $\hat{p}$ ), enquanto que seu lado direito envolve apenas um operador limitado (1), o que suscita de imediato um problema relativo à solidez matemática daquela afirmação básica da MQ.

Para contornar tal dificuldade, Weyl propôs a substituição da relação de comutação (1) por uma nova, mantendo ainda a essência da não comutatividade. Esta nova proposta envolve basicamente operadores unitários escritos em termos dos operadores coordenada e momento

\footnotetext{
${ }^{10} \mathrm{~A}$ acomodação matemática dessa classe de operadores aos fundamentos propostos para o formalismo da MQ por Dirac 20], levou a uma abordagem que ganhou significância e aceitação na comunidade dos físicos, mas também gerou inicialmente algum desconforto entre os matemáticos 21].
} 
linear 11

$$
\hat{U}(\alpha)=\exp \left(\frac{\mathrm{i}}{\hbar} \alpha \hat{p}\right) \quad \text { e } \hat{V}(\beta)=\exp \left(\frac{\mathrm{i}}{\hbar} \beta \hat{q}\right)
$$

sendo a relação de comutação entre eles dada por

$$
\hat{U}(\alpha) \hat{V}(\beta)=\exp \left(\frac{\mathrm{i}}{\hbar} \alpha \beta\right) \hat{V}(\beta) \hat{U}(\alpha) .
$$

Já que a solução encontrada envolve somente operadores unitários - que por sua vez são limitados e definidos em todo o espaço -, a objeção original seria assim resolvida.

Antes de prosseguirmos, um breve hiato é interessante para estabelecer algumas propriedades matemáticas simples para os operadores unitários, que permitem, em princípio, melhor entender o resultado de suas ações sobre os autovetores $|q\rangle$ e $|p\rangle$. Por exemplo,

$$
\hat{U}(\alpha)|q\rangle=|q-\alpha\rangle \quad \text { e } \quad \hat{V}(\beta)|p\rangle=|p+\beta\rangle
$$

representam deslocamentos nos eixos coordenados correspondentes, enquanto que

$\hat{U}(\alpha)|p\rangle=\exp \left(\frac{\mathrm{i}}{\hbar} \alpha p\right)|p\rangle \quad$ e $\quad \hat{V}(\beta)|q\rangle=\exp \left(\frac{\mathrm{i}}{\hbar} \beta q\right)|q\rangle$

descrevem equações de autovalores. Uma vez que as bases $\{|q\rangle\}$ e $\{|p\rangle\}$ são complementares, com seu kernel de Fourier dado por $\langle q \mid p\rangle=\frac{1}{\sqrt{2 \pi}} \exp \left(\frac{\mathrm{i}}{\hbar} q p\right)$, tais deslocamentos admitem decomposições de Fourier específicas para cada caso, a saber:

$$
\begin{aligned}
& |q-\alpha\rangle=\int_{\mathbb{R}} \frac{d p}{\sqrt{2 \pi}} \exp \left[-\frac{\mathrm{i}}{\hbar}(q-\alpha) p\right]|p\rangle, \\
& |p+\beta\rangle=\int_{\mathbb{R}} \frac{d q}{\sqrt{2 \pi}} \exp \left[\frac{\mathrm{i}}{\hbar}(p+\beta) q\right]|q\rangle .
\end{aligned}
$$

Esses resultados preliminares podem aqui ser interpretados como um prelúdio para a futura descrição da $\mathrm{MQ}$ no espaço de fases [41].

O problema abordado por Weyl foi retomado por von Neumann posteriormente, a partir da equação (3) para os operadores unitários, com o intuito de provar que as soluções irredutívei: ${ }^{12}$ desta são necessariamente as soluções da relação de comutação (1). Refraseando, as soluções encontradas para a equação (3) resolvem o problema da inconsistência matemática aparente na relação de comutação (11) para os operadores hermitianos coordenada e momento linear.

Como ponto de partida inicial para tal demonstração, von Neumann [4] considerou uma família de operadores unitários uniparamétricos coincidentes com a prescrição de Weyl - vide equação (2) -, de forma a explicitar suas regras de multiplicação

$$
\begin{aligned}
& \hat{U}(\alpha) \hat{U}\left(\alpha^{\prime}\right)=\hat{U}\left(\alpha+\alpha^{\prime}\right) \quad\left(\alpha, \alpha^{\prime} \in \mathbb{R}\right), \\
& \hat{V}(\beta) \hat{V}\left(\beta^{\prime}\right)=\hat{V}\left(\beta+\beta^{\prime}\right) \quad\left(\beta, \beta^{\prime} \in \mathbb{R}\right),
\end{aligned}
$$

\footnotetext{
${ }^{11}$ Deve-se observar que as dimensões de $\alpha$ e $\beta$ são, respectivamente, coordenada e momento linear, $\operatorname{com} \alpha, \beta \in \mathbb{R}$.

${ }^{12}$ Ver Nota 7 da tradução do artigo de von Neumann para o entendimento do que são soluções irredutíveis para operadores (no presente caso, operadores unitários) - consultar também referência 22 .
}

bem como ressaltar o caráter não comutativo de seus elementos, aqui representado pela equação $(3),{ }^{13}$ A solução procurada consiste então no conjunto de todos os operadores com estas propriedades, o que deve ser determinado a posteriori. Como recurso auxiliar para a prova, von Neumann introduziu ainda uma outra família de operadores unitários ${ }^{14}$

$$
\begin{aligned}
\hat{S}(\alpha, \beta) & =\exp \left(-\frac{\mathrm{i}}{2 \hbar} \alpha \beta\right) \hat{U}(\alpha) \hat{V}(\beta) \\
& =\exp \left(\frac{\mathrm{i}}{2 \hbar} \alpha \beta\right) \hat{V}(\beta) \hat{U}(\alpha)
\end{aligned}
$$

tal que, para eles, vale a lei de composiçã ${ }^{15}$

$$
\hat{S}(\alpha, \beta) \hat{S}\left(\alpha^{\prime}, \beta^{\prime}\right)=\exp \left[\frac{\mathrm{i}}{2 \hbar}\left(\alpha \beta^{\prime}-\beta \alpha^{\prime}\right)\right] \hat{S}\left(\alpha+\alpha^{\prime}, \beta+\beta^{\prime}\right),
$$

bem como a operação traço

$$
\operatorname{Tr}\left[\hat{S}(\alpha, \beta) \hat{S}^{\dagger}\left(\alpha^{\prime}, \beta^{\prime}\right)\right]=2 \pi \hbar \delta\left(\alpha-\alpha^{\prime}\right) \delta\left(\beta-\beta^{\prime}\right) .
$$

Convém salientar que von Neumann não menciona em nenhum momento que $\{\hat{S}(\alpha, \beta): \alpha, \beta \in \mathbb{R}\}$ constitui uma base de operadores no espaço de Hilbert, apesar de utilizá-la de forma explícita para seus propósitos.

A partir deste conjunto básico de operadores é possível então escrever combinações lineares

$$
\hat{A}=\iint_{\mathbb{R}^{2}} \mathbf{a}(\alpha, \beta) \hat{S}(\alpha, \beta) d \alpha d \beta,
$$

onde $\mathbf{a}(\alpha, \beta)$ representa uma função absolutamente integrável (chamada núcleo do operador $\hat{A}$ ), sendo a integral acima absolutamente convergente. Consequentemente, a ação de um tal operador linear $\hat{A}$ sobre funções $f$ e $g$ de um espaço de Hilbert pode ser prontamente determinada através do valor esperado

$$
\operatorname{Tr}[\hat{A}|f\rangle\langle g|]=\iint_{\mathbb{R}^{2}} \mathbf{a}(\alpha, \beta) \operatorname{Tr}[\hat{S}(\alpha, \beta)|f\rangle\langle g|] d \alpha d \beta .
$$

${ }^{13}$ Segundo Weyl [40], o conjunto das relações de composição e comutação permite caracterizar a estrutura cinemática de um sistema quântico como um grupo abeliano irredutível de rotações com raio unitário.

${ }^{14} \mathrm{~A}$ escolha de parâmetros $\alpha=q$ e $\beta=-p$ faz com que a família de operadores unitários $\hat{S}(q,-p)$ coincida exatamente com aquela de operadores deslocamentos $\hat{D}(q, p):=\exp \left[\frac{\mathrm{i}}{\hbar}(q \hat{p}-p \hat{q})\right]$, sendo estes operadores muito utilizados em Óptica Quântica no estudo de representações associadas aos estados coerentes 46 49. De fato, ao adotarmos o estado de vácuo $|0\rangle$ como um estado de referência, a ação $\hat{S}(q,-p)|0\rangle \equiv|q, p\rangle$ permite definir um conjunto de estados coerentes indexados, neste caso, pelas variáveis posição e momentum linear. É interessante observar que von Neumann, ao estabelecer a família de operadores unitários 5 , torna-se indiretamente, nesse preciso momento, um precursor das representações de estados coerentes.

${ }^{15}$ Os operadores $\hat{S}^{\dagger}(\alpha, \beta)=\hat{S}(-\alpha,-\beta)$ (adjunto) e $\hat{S}(0,0)=\hat{1}$ (identidade) decorrem das propriedades dos operadores unitários parametrizados previamente definidos; além disso, note-se que

$$
\hat{S}^{\dagger}(\alpha, \beta) \hat{S}(\alpha, \beta)=\hat{S}(\alpha, \beta) \hat{S}^{\dagger}(\alpha, \beta)=\hat{1} .
$$


Além disso, por intermédio da equação (7) obtém-se, após alguns cálculos, uma expressão para $\mathbf{a}(\alpha, \beta)$ conforme segue: 16

$$
\mathbf{a}(\alpha, \beta)=(2 \pi \hbar)^{-1} \operatorname{Tr}\left[\hat{A} \hat{S}^{\dagger}(\alpha, \beta)\right] .
$$

De posse do conjunto de operadores unitários $\hat{S}(\alpha, \beta)$ e suas respectivas propriedades, certos resultados emergem imediatamente:

- $x \hat{A}$ (sendo $x$ um escalar) tem núcleo $x \mathbf{a}(\alpha, \beta)$;

- $\hat{A}^{\dagger}$ possui núcleo $[\mathbf{a}(-\alpha,-\beta)]^{*}$;

- se $\hat{A}$ e $\hat{B}$ têm núcleos $\mathbf{a}(\alpha, \beta)$ e $\mathbf{b}(\alpha, \beta)$, respectivamente, então $\hat{A}+\hat{B}$ tem núcleo $\mathbf{a}(\alpha, \beta)+\mathbf{b}(\alpha, \beta)$;

- os núcleos associados aos operadores posição e momentum linear são diretamente proporcionais a derivadas de funções delta, isto é, tais operadores não possuem núcleos bem comportados e refletem assim os problemas inerentes ao par canônico $\hat{q}$ e $\hat{p}$

- em contrapartida, os projetores $|q\rangle\langle q|$ e $|p\rangle\langle p|$ possuem núcleos bem comportados uma vez que ambos os núcleos são proporcionais a funções delta.

Contudo, o cálculo do núcleo relacionado ao produto $\hat{A} \hat{B}$ é menos intuitivo por envolver fases adicionais nas composições dos operadores $\hat{S}(\alpha, \beta)$, como pode ser visto na equação (6). De forma explícita, o resultado para o núcleo do operador $\hat{A} \hat{B}$ é uma convolução dos respectivos núcleos $\mathbf{a}(\alpha, \beta)$ e $\mathbf{b}(\alpha, \beta)$, ou seja,

$$
\hat{A} \hat{B}=\iint_{\mathbb{R}^{2}} \mathbf{F}(\alpha, \beta) \hat{S}(\alpha, \beta) d \alpha d \beta,
$$

com o núcleo $\mathbf{F}(\alpha, \beta)$ dado por

$$
\iint_{\mathbb{R}^{2}} \mathrm{e}^{\frac{\mathrm{i}}{2 \hbar}\left(\alpha \beta^{\prime}-\beta \alpha^{\prime}\right)} \mathbf{a}\left(\alpha-\alpha^{\prime}, \beta-\beta^{\prime}\right) \mathbf{b}\left(\alpha^{\prime}, \beta^{\prime}\right) d \alpha^{\prime} d \beta^{\prime} .
$$

Então, um produto escalar envolvendo o produto dos operadores $\hat{A} \hat{B}$ e funções $f$ e $g$ do espaço de Hilbert, pode também ser entendido como

$$
\operatorname{Tr}[\hat{A} \hat{B}|f\rangle\langle g|]=\iint_{\mathbb{R}^{2}} \mathbf{F}(\alpha, \beta) \operatorname{Tr}[\hat{S}(\alpha, \beta)|f\rangle\langle g|] d \alpha d \beta .
$$

Para provar que todos os operadores podem ser escritos nessa forma - em termos de seus núcleos - von Neumann provou também que se $\hat{A}$ for o operador nulo, seu núcleo é igual a 0 .

Por fim, von Neumann provou rigorosamente que uma família de operadores unitários $\hat{U}(\alpha)$ e $\hat{V}(\beta)$ é univocamente definida se, a menos de transformações unitárias, ela satisfizer as regras de multiplicação de Weyl - vide

\footnotetext{
${ }^{16}$ Convém mencionar que ambas as equações 77 e $\sqrt{9}$ não foram derivadas explicitamente por von Neumann, mas sim por Groenewold 43], e são aqui apresentadas exclusivamente para facilitar o bom entendimento do leitor.
}

equação (3). A composição dos elementos daquela família permite então definir um conjunto de operadores $\hat{S}(\alpha, \beta)$ cujo domínio varre todo o espaço de Hilbert; consequentemente, suas combinações lineares permitem decompor um operador $\hat{A}$ de uma maneira bem comportada, já que os coeficientes desta decomposição são absolutamente integráveis ${ }^{17}$ Uma vez fundamentados todos os passos supracitados, von Neumann [4] parte para a solução do problema da unicidade da relação de comutação (1), o que ele faz com pleno sucess ${ }^{18}$ (os procedimentos adotados para a demonstração comprovam sua caraterística própria e única de raciocício matemático para a época).

\section{Conexões com a Descrição da MQ no Espaço de Fases}

Ao longo das décadas de 1930 e 1940 foi desenvolvida uma importante descrição da MQ baseada na concepção de um espaço de fases em moldes próximos daquele concebido pela Mecânica Clássica [1, 2], embora adaptado para dar conta das características próprias da teoria quântica. Nesta nova abordagem, tem-se basicamente que a cada operador está associada uma única função de variáveis canônicas clássicas, que se compõem com regras próprias; por exemplo, ao operador densidade associa-se a função de Wigner, $\hat{\rho} \rightarrow W(q, p)$, e aos operadores posição e momento linear estão associadas as funções,

$$
\hat{q} \rightarrow q \quad \text { e } \quad \hat{p} \rightarrow p
$$

Por conseguinte, a partir da função de Wigner, $W(q, p)$, e de funções associadas a variáveis dinâmicas, obtêm-se resultados para valores médios em completa conexão com o conjunto de valores possíveis de medidas para aquela variável. Com isso, observa-se que o procedimento matemático de associação operador $\rightarrow$ função desempenha um papel essencial no formalismo WWGM [41].

A correspondência entre as formulações de Schrödinger e WWGM, previamente discutidas ao longo do texto, foi refinada por Snygg [50], que estabeleceu novas substituições dos operadores posição e momento linear por suas representações diferenciais específicas correspondentes, ou seja,

$$
\hat{q} \rightarrow q-\frac{h}{2 \mathrm{i}} \frac{\partial}{\partial p} \quad \text { e } \quad \hat{p} \rightarrow p+\frac{h}{2 \mathrm{i}} \frac{\partial}{\partial q} .
$$

O limite clássico, obtido como $h \rightarrow 0$, também é estudado neste contexto.

Antes de prosseguirmos com a discussão acerca da associação operador $\rightarrow$ função, mencionada no parágrafo inicial, convém estabelecer uma conexão explícita entre

\footnotetext{
${ }^{17}$ Em suma, os operadores unitários $\hat{U}(\alpha)$ e $\hat{V}(\beta)$, com sua relação de comutação, fundamentam uma descrição da MQ de um sistema físico com um grau de liberdade. Porém, esta abordagem não será adequada para sistemas com infinitos graus de liberdade, como em teorias de campos.

${ }^{18}$ Para uma versão detalhada e rigorosa desta prova, realizada posteriormente ao trabalho de von Neumann, ver referência 22.
} 
os resultados obtidos no trabalho de von Neumann [4] e aqueles relacionados ao formalismo WWGM. Para tanto, iremos apresentar uma primeira propriedade importante relacionada à conexão (não-trivial) entre o operador adjunto $\hat{S}^{\dagger}(\alpha, \beta)$ e o operador paridade $\hat{\mathcal{P}} 51,52$,

$$
\iint_{\mathbb{R}^{2}} \frac{d \alpha d \beta}{2 \pi \hbar} \hat{S}^{\dagger}(\alpha, \beta)=2 \int_{\mathbb{R}} d q|q\rangle\langle-q|=2 \hat{\mathcal{P}} .
$$

Em seguida, ao fazermos uma transformação unitária específica na equação acima, obtemos uma expressão exatamente igual àquela da base de operadores de WeylWigner ${ }^{19}$

$$
\hat{\Delta}(q, p)=\iint_{\mathbb{R}^{2}} \frac{d \alpha d \beta}{2 \pi \hbar} \exp \left\{\frac{\mathrm{i}}{\hbar}[\beta(q-\hat{q})+\alpha(p-\hat{p})]\right\}
$$

no formalismo WWGM, mediante uma escolha adequada de parâmetros. Assim, ao realizarmos este procedimento mediante o auxílio do operador unitário $\hat{S}(q,-p)$, chegamos finalmente à relação desejada 52

$$
\hat{\Delta}(q, p)=\hat{S}^{\dagger}(q,-p)(2 \hat{\mathcal{P}}) \hat{S}(q,-p) .
$$

Esta identidade demonstra, num primeiro momento, que a família de operadores unitários (5), introduzida por von Neumann, tem um papel fundamental neste processo ao estabelecer uma conexão formal com o operador paridade $\hat{\mathcal{P}}$ e a base de operadores $\hat{\Delta}(q, p)$ de Weyl-Wigner; num segundo momento, a equação $\sqrt{12}$ nos leva diretamente ao cálculo da função de Wigner, $W(q, p)=\operatorname{Tr}[\hat{\rho} \hat{\Delta}(q, p)]$, para um dado sistema físico ${ }^{20}$ Nesse sentido, os resultados obtidos por von Neumann em sua demonstração da unicidade dos operadores unitários representam um embrião de suma importância para os fundamentos teóricos da MQ em sua descrição no espaço de fases.

A transformação unitária que permite conectar a base de operadores $\hat{\Delta}(q, p)$ e o operador paridade $\hat{\mathcal{P}}$, também nos leva a mostrar que essa base e $\{\hat{S}(\alpha, \beta): \alpha, \beta \in \mathbb{R}\}$ se encontram associadas por uma transformada de Fourier,

$$
\hat{\Delta}(q, p)=\iint_{\mathbb{R}^{2}} \frac{d \alpha d \beta}{2 \pi \hbar} \exp \left[\frac{\mathrm{i}}{\hbar}(\alpha p+\beta q)\right] \hat{S}^{\dagger}(\alpha, \beta) .
$$

Esta expressão reflete fielmente a relação existente entre a função de Wigner e sua função característica

$$
\widetilde{W}(\alpha, \beta)=\operatorname{Tr}\left[\hat{\rho} \hat{S}^{\dagger}(\alpha, \beta)\right] .
$$

Retornando à discussão inicial, embora Weyl 440 tenha proposto uma associação função $\rightarrow$ operador 21 ele

\footnotetext{
19 Deve ser observado que esta equação corresponde a uma escolha particular e não única do ponto de partida, tendo em vista que $\hat{\Delta}(q, p)$ admite representações diferentes 41 .

${ }^{20}$ É importante identificar que $\hat{\Delta}(q, p)$ também pode ser interpretado como um operador de reflexão no espaço de fase no entorno do ponto $(q, p)$ 51 53. De fato, em conjunto com os operadores de deslocamentos $\hat{S}(q,-p)$, eles definem um grupo maior: o grupo afim quântico. Logo, obtém-se um par de representações equivalentes neste espaço de fases que estão associadas a operadores unitários de reflexões ou deslocamentos.

${ }^{21}$ Esta proposta não é uma regra de quantização; ela de fato põe em correspondência um operador a uma função de variáveis clássicas, não o contrário.
}

não estabeleceu um procedimento correspondente para o produto de dois ou mais operadores. O trabalho de von Neumann [4] preenche exatamente esta lacuna deixada por Weyl e traz em seu bojo uma contribuição de caráter matemático importante para o pronto estabelecimento de regras claras e precisas para a associação entre produtos de operadores e funções de variáveis canônicas clássicas. A expressão 10 para o núcleo $\mathbf{F}(\alpha, \beta)$ exemplifica de forma nítida essa regra de associação, que no caso em particular se refere ao produto $\hat{A} \hat{B}$ de dois operadores e seus correspondentes núcleos $\mathbf{a}(\alpha, \beta)$ e $\mathbf{b}(\alpha, \beta)$. Portanto, da abordagem de von Neumann depreendem-se duas conclusões:

- se $\{\hat{S}(\alpha, \beta): \alpha, \beta \in \mathbb{R}\}$ é um conjunto de operadores com suas propriedades definidas pelas equações (6) e (7), os núcleos dos operadores são as funções associadas aos operadores através da equação (9);

- a expressão definidora da função associada ao produto de dois operadores, equação 10, não envolve o produto das funções associadas aos operadores, mas sim uma convolução delas. Este resultado é fundamental quando se calculam as expressões associadas a comutadores e anticomutadores.

Em particular, esta forma de associação está intimamente relacionada ao que é conhecido como produto- $\star$ na descrição da MQ no espaço de fases ${ }^{22}$

Relativamente ao procedimento de associação, é digno de nota que a equação (8) pode ser interpretada alternativamente como correspondendo à decomposição, com regras próprias e bem definidas, de um operador linear $\hat{A}$ em um conjunto de operadores $\hat{S}(\alpha, \beta)$ (neste contexto, este conjunto representa uma base de operadores no espaço de Hilbert). Essa hipótese é constatada ao longo do trabalho, embora von Neumann não a tenha afirmado nem provado explicitamente, mas a tenha utilizado.

von Neumann não teve como objetivo primordial estabelecer a regra de associação entre operadores e funções de variáveis canônicas clássicas; seu trabalho, contudo, serviu como ponto de partida para Groenewold fundamentar a descrição da MQ no espaço de fases. No trabalho original de Groenewold [43], a ideia de uma associação operador $\rightarrow$ função de variáveis canônicas clássicas voltou a ser de interesse central. Para construir tal associação, o ponto fundamental da abordagem de Groenewold consistia na procura de um conjunto de operadores com o qual seria possível expandir qualquer observável de interesse físico. O resultado desta busca foi o conjunto de operadores unitários $\hat{S}(\alpha, \beta)$ previamente discutido por von Neumann.

Com base nessa abordagem com operadores unitários, a questão matemática referente à associação inversa, isto é, função de variáveis canônicas clássicas $\rightarrow$ operadores, que von Neumann [4] não abordou, também mereceu atenção

\footnotetext{
${ }^{22} \mathrm{Na}$ verdade, ela é a transformada de Fourier do que é usualmente conhecido como produto- $\star$, cuja forma diferencial se encontra deduzida em detalhes na referência 41.
} 
e foi estudada por Groenewold [43]: certas funções estarão associadas a operadores se satisfizerem a condição básica (9). Tal condição restritiva mostra então que não serão todas as funções de variáveis canônicas clássicas que estarão associadas a operadores.

Partindo de um enfoque diferente e baseado em conceitos puramente estatísticos, Moyal [44] chegou aos mesmos resultados de Groenewold. Sua expressão para a relação de comutação de dois operadores - em termos das funções a eles associadas - é a mesma, bem como a expressão para a evolução temporal do estado do sistema físico ${ }^{23}$

Com esta importante compilação de resultados, a proposta de descrição da Mecânica Quântica no espaço de fases ganhava força e corpo do ponto de vista teórico e também estava muito bem fundamentada [1,2]. Relações de comutação para operadores - essenciais na MQ - podem agora ser vertidas para a linguagem de funções de variáveis canônicas clássicas, sendo que a operação básica entre aquelas funções é o produto- $\star$, como antecipado originalmente por von Neumann [4]. Da mesma forma, valores médios de observáveis físicos são obtidos na medida em que o estado do sistema quântico é mapeado no espaço de fases via função de Wigner. Neste contexto, é digno de nota mencionar brevemente o fato de que esta função pode assumir valores negativos; caso isto ocorra, esta característica está relacionada estritamente à natureza não-clássica do estado do sistema físico sob investigação ${ }^{24}$ o que não impede que valores médios sejam precisamente calculados e reflitam valores mensuráveis em laboratório.

\section{Considerações Finais}

Existem diversas formulações da MQ aceitas hoje em dia na literatura 62]. Na seção2 apresentamos as formulações via função de onda, matricial, integral de trajetória e espaço de fases, por razões históricas e por terem pontos em comum. A ênfase adotada na descrição da MQ no espaço de fases se faz necessária neste particular caso devido ao seu completo entrelaçamento com o trabalho de von Neumann.

O formalismo da MQ no espaço de fases é uma ferramenta matemática concreta e extremamente útil para a investigação de diversos fenômenos físicos [1,2]; suas bases foram lançadas no conjunto de resultados matemáticos relacionados ao trabalho de von Neumann de 1931, o qual apresenta um arcabouço teórico embrionário para a concepção de um espaço de fases genuinamente quântico. É digno de observação o fato de que von Neumann 4 não expôs o procedimento matemático associado à obtenção do núcleo do operador, embora pudesse tê-lo feito, além de não ter mencionado que os núcleos dos

\footnotetext{
${ }^{23} \overline{E m}$ particular, trata-se de uma equação integro-diferencial e é conhecida na literatura como equação dinâmica de Moyal 44 45].

${ }^{24}$ Embora seja denominada tecnicamente como função distribuição de quase-probabilidades, por ter os axiomas de Kolmogorov para teoria de probabilidades relaxados, a função de Wigner apresenta uma ampla gama de aplicações em física 54 61.
}

operadores os representam num espaço de fases dual, pois estão interligados por uma transformada de Fourier. Todas estas inferências foram feitas a posteriori através de considerações adicionais àquelas por ele usadas, uma vez que as fez somente para mostrar que o conjunto de operadores $\hat{S}(\alpha, \beta)$ tem como domínio todo o espaço de Hilbert. Neste sentido, conclui-se que von Neumann não é um proponente da descrição da MQ no espaço de fases. Não obstante, este seu trabalho estabeleceu pela primeira vez os fundamentos da associação operador $\rightarrow$ função de variáveis canônicas clássicas em bases matematicamente sólidas; em particular, ele foi o primeiro a descrever como seria a associação de produtos de operadores e a exibir a convolução necessária para tal, usando para tanto um conjunto completo de operadores, estendendo assim a abordagem inicial de Weyl.

O procedimento matemático delineado no trabalho de von Neumann também traz importantes elementos precursores das representações de estados coerentes, sendo estas representações capítulo obrigatório em livros textos sobre Óptica Quântica. Não obstante ao fato do autor trabalhar somente com operadores unitários e não mencionar em nenhum momento o estado de vácuo, a simples identificação em (5) dos parâmetros $\alpha=q$ e $\beta=-p$, faz com que aquela família de operadores unitários $\hat{S}(q,-p)$ coincida exatamente com os geradores de deslocamentos $\hat{D}(q, p)$ unitários, geradores estes amplamente utilizados na definição de estados coerentes [46 49].

Finalmente, resta-nos sugerir a inclusão das iniciais de von Neumann à sigla WWGM, passando desta forma a designar o formalismo da MQ no espaço de fases por WvNWGM.

\section{Agradecimentos}

Os autores gostariam de agradecer as colaborações dos árbitros por terem apontado questões pontuais relevantes ao par complementar de textos 'tradução' (material suplementar) e 'comentários', as quais possibilitaram melhoras significativas quanto ao rigor e abrangência para ambas as versões finais.

\section{Material Suplementar}

O seguinte material suplementar está disponível online:

Material Suplementar - A unicidade dos operadores de Schrödinger

\section{Referências}

[1] T.L. Curtright, D.B. Fairlie e C.K. Zachos, A Concise Treatise on Quantum Mechanics in Phase Space (World Scientific, Singapore, 2014).

[2] C.K. Zachos, D.B. Fairlie e T.L. Curtright, Quantum Mechanics in Phase Space: An Overview with Selected Papers (World Scientific, New Jersey, 2005).

[3] L. van Hove, Bull. Amer. Math. Soc. 64, 95 (1958). 
[4] J. von Neumann, Math. Ann. 104, 570 (1931).

[5] M. Jammer, The conceptual development of quantum mechanics (McGraw-Hill, New York, 1966).

[6] B.L. van der Waerden, Sources of Quantum Mechanics (Dover Publications, New York, 2007).

[7] J. Mehra e H. Rechenberg, The Quantum Theory of Planck, Einstein, Bohr and Sommerfeld: Its Foundation and the Rise of Its Difficulties 1900-1925 (SpringerVerlag, New York, 1982), v. 1.

[8] J. Mehra e H. Rechenberg, The Discovery of Quantum Mechanics, 1925 (Springer-Verlag, New York, 1982), v. 2.

[9] J. Mehra e H. Rechenberg, The Formulation of Matrix Mechanics and Its Modifications, 1925-1926 (SpringerVerlag, New York, 1982), v. 3.

[10] J. Mehra e H. Rechenberg, The Reception of the New Quantum Mechanics, 1925-1926 (Springer-Verlag, New York, 1982), v. 4.

[11] J. Mehra e H. Rechenberg, The Creation of Wave Mechanics; Early Response and Applications, 1925-1926 (Springer-Verlag, New York, 1982), v. 5.

[12] J. Mehra e H. Rechenberg, The Probability Interpretation and the Statistical Transformation Theory, the Physical Interpretation, and the Empirical and Mathematical Foundations of Quantum Mechanics, 1926-1932, (Springer-Verlag, New York, 1982).

[13] M. de Gosson, The principles of Newtonian and quantum mechanics: The need for Planck's constant, $h$ (Imperial College Press, London, 2001).

[14] M. de Gosson, Symplectic Geometry and Quantum Mechanics (Birkhäuser Verlag, Berlin, 2006).

[15] W. Heisenberg, Z. Physik 33, 879 (1925).

[16] M. Born e P. Jordan, Z. Physik 34, 858 (1925).

[17] M. Born, W. Heisenberg e P. Jordan, Z. Physik 35, 557 (1925).

[18] M. Born, Z. Physik 37, 863 (1926).

[19] M. Born, Z. Physik 38, 803 (1926).

[20] P.A.M. Dirac, The Principles of Quantum Mechanics (Oxford Science Publications, New York, 1993).

[21] J. von Neumann, Mathematical Foundations of Quantum Mechanics (Princeton University Press, Princeton, 1971).

[22] E. Prugovečki, Quantum Mechanics in Hilbert Space (Academic Press, New York, 1981).

[23] A.N. Kolmogorov e S.V. Fomin, Elements of the Theory of Functions and Functional Analysis (Dover Publications, New York, 1999).

[24] C.R. Oliveira, Introdução à Análise Funcional (IMPA, Rio de Janeiro, 2015).

[25] N.A. Lemos, Convite à Física Matemática (Editora Livraria da Física, São Paulo, 2013).

[26] W. Heisenberg, Z. Physik 43, 172 (1927).

[27] E. Schrödinger, Ann. Physik 384, 361 (1926).

[28] R. Eisberg e R. Resnick, Quantum Physics of Atoms, Molecules, Solids, Nuclei, and Particles (John Wiley \& Sons, New York, 1985).

[29] F. Caruso e V. Oguri, Física Moderna: Origens Clássicas e Fundamentos Quânticos (Editora Campus, Rio de Janeiro, 2006).

[30] P.A. Tipler e R.A. Llewellyn, Física Moderna (LTC, Rio de Janeiro, 2010).

[31] E. Schrödinger, Ann. Physik 384, 734 (1926).
[32] C. Eckart, Proceedings of the National Academy of Sciences of the United States of America 12, 473 (1926).

[33] P.A.M. Dirac, Physik. Zeits. Sowjetunion 3, 64 (1933).

[34] P.A.M. Dirac, Rev. Mod. Phys. 17, 195 (1945).

[35] J. Schwinger, Selected Papers on Quantum Electrodynamics (Dover Publications, New York, 2012).

[36] M.A.M. de Aguiar, Tópicos de Mecânica Clássica (Editora Livraria da Física, São Paulo, 2011), v. 15.

[37] R.P. Feynman, Rev. Mod. Phys. 20, 367 (1948).

[38] I.H. Duru e H. Kleinert, Phys. Lett. B 84, 185 (1979).

[39] H. Kleinert, Path Integrals in Quantum Mechanics, Statistics, Polymer Physics, and Financial Markets (World Scientific, Singapore, 2009).

[40] H. Weyl, The Theory of Groups and Quantum Mechanics (Dover Publications, New York, 1950).

[41] M.A. Marchiolli, Rev. Bras. Ens. Fís. 24, 421 (2002).

[42] E. Wigner, Phys. Rev. 40, 749 (1932).

[43] H. Groenewold, Physica 12, 405 (1946).

[44] J. Moyal, Proc. Camb. Phil. Soc. 45, 99 (1949).

[45] M. Bartlett e J. Moyal, Proc. Camb. Phil. Soc. 45, 545 (1949).

[46] W.H. Louisell, Quantum Statistical Properties of Radiation (John Wiley \& Sons, New York, 1973).

[47] A. Perelomov, Generalized Coherent States and Their Applications (Springer-Verlag, Berlin, 1986).

[48] W.P. Schleich, Quantum Optics in Phase Space (WileyVCH, Berlin, 2001).

[49] C.C. Gerry e P.L. Knight, Introductory Quantum Optics (Cambridge University Press, Cambridge, 2006).

[50] J. Snygg, Am. J. Phys. 48, 964 (1980).

[51] A. Grossmann, Commun. Math. Phys. 48, 191 (1976).

[52] A. Royer, Phys. Rev. A 15, 449 (1977).

[53] A.M.O. Almeida, Phys. Rep. 295, 265 (1998).

[54] E.J. Heller, J. Chem. Phys. 65, 1289 (1976).

[55] E.J. Heller, J. Chem. Phys. 67, 3339 (1977).

[56] P. Carruthers e F. Zachariasen, Rev. Mod. Phys. 55, 245 (1983).

[57] M. Hillery, R.F. O'Connell, M.O. Scully e E.P. Wigner, Phys. Rep. 106, 121 (1984).

[58] H-W. Lee, Phys. Rep. 259, 147 (1995).

[59] L.G. Lutterbach e L. Davidovich, Phys. Rev. Lett. 78, 2547 (1997).

[60] M. Belloni, M.A. Doncheski e R.W. Robinett, Am. J. Phys. 72, 1183 (2004)

[61] W.B. Case, Am. J. Phys. 76, 937 (2008).

[62] D.F. Styer, M.S. Balkin, K.M. Becker, M.R. Burns, C.E. Dudley, S.T. Forth, J.S. Gaumer, M.A. Kramer, D.C. Oertel, L.H. Park et al., Am. J. Phys. 70, 288 (2002). 